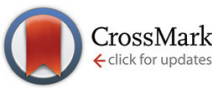

Cite this: Biomater. Sci., 2015, 3, 581

Received 28th November 2014

Accepted 8th January 2015

DOI: 10.1039/c4bm00411f

www.rsc.org/biomaterialsscience

\section{A novel hanging spherical drop system for the generation of cellular spheroids and high throughput combinatorial drug screening $\dagger$}

\author{
A. I. Neto, ${ }^{a, b}$ C. R. Correia, ${ }^{a, b}$ M. B. Oliveira, ${ }^{a, b}$ M. I. Rial-Hermida, ${ }^{c}$ C. Alvarez-Lorenzo, ${ }^{c}$ \\ R. L. Reis ${ }^{a, b}$ and J. F. Mano*a,b
}

We propose a novel hanging spherical drop system for anchoring arrays of droplets of cell suspension based on the use of biomimetic superhydrophobic flat substrates, with controlled positional adhesion and minimum contact with a solid substrate. By facing down the platform, it was possible to generate independent spheroid bodies in a high throughput manner, in order to mimic in vivo tumour models on the lab-on-chip scale. To validate this system for drug screening purposes, the toxicity of the anti-cancer drug doxorubicin in cell spheroids was tested and compared to cells in 2D culture. The advantages presented by this platform, such as feasibility of the system and the ability to control the size uniformity of the spheroid, emphasize its potential to be used as a new low cost toolbox for high-throughput drug screening and in cell or tissue engineering.

Cell-based high throughput screening (HTS) has been proposed as a powerful tool for rapid identification of drug candidates from large biological and chemical libraries with desired efficacy, which can accelerate drug discovery. ${ }^{1-4}$ Most studies of cell and tissue regulation have relied on the analysis of cell behaviour in two-dimensional (2D) cell culture models. However, the 2D model fails to simulate the in vivo cellular microenvironment, thus compromising cellular functions, namely metabolic activity, proliferation, differentiation and cell death. Efforts to address these limitations led to the development of three-dimensional (3D) cell culture models in which cells are grown within an engineered extracellular matrix (ECM). ${ }^{5}$ HTS of anticancer drugs is usually performed using high-density multi-well plates with high-level and expensive robotic devices for cell seeding and drug management. ${ }^{6-12}$ Recent advances in $3 \mathrm{D}$ cancer cell culture

\footnotetext{
${ }^{a} 3 B$ 's Research Group - Biomaterials, Biodegradables and Biomimetics, University of Minho, AvePark, 4806-90 Taipas, Guimarães, Portugal

${ }^{b}$ ICVS/3B'sPT Government Associate Laboratory, Braga/Guimarães, Portugal.

E-mail:jmano@dep.uminho.pt

${ }^{c}$ Department Farmacia y Tecnología Farmacéutica, Universidad de Santiago de

Compostela, 15782-Santiago de Compostela, Spain

$\dagger$ Electronic supplementary information (ESI) available: Experimental section. See DOI: $10.1039 / \mathrm{c} 4 \mathrm{bm} 00411 \mathrm{f}$
}

have used cellular spheroids as an adequate model, exhibiting a complex microenvironment similar to tumours in vivo and comparable drug responses. ${ }^{13-18}$ Spheroids are self-assembled spherical clusters of cell colonies cultured in environments where cell-cell interactions dominate over cell-substrate interactions, and they naturally mimic avascular tumours with inherent metabolic (dependent on oxygen) and proliferative (dependent on nutrients) gradients. ${ }^{19}$

3D spheroids can be easily produced by various simple methods, namely hanging drop cultures, engineered microfabrication of round-bottom and non-adherent platforms, microfluidic devices, spinner flasks, NASA rotary culture, thermo-responsible substrates and $3 \mathrm{D}$ scaffolds. ${ }^{20}$ In particular, the hanging drop cultures offer an accessible and reliable method for generating higher numbers of spheroids with a precise and homogeneous size control and suitable compatibility for high throughput assays. Current developments in microarray devices are directed towards further reductions in sample volumes, increasing analytical throughput and integration of pre- and post-assay processing. Innovative technologies enable, for example, 384 spheroids to be cultured in a hanging drop fashion in the same platform. ${ }^{21}$ However, the production and utilization of such innovative platforms require high precision and sophisticated equipment that can be extremely expensive.

In our group, we made advances in this field, creating an innovative low-cost platform for individually dispensing miniaturized biomaterials, cells and culture medium, based on the use of patterned superhydrophobic (SH) surfaces. $^{22-26}$ The topography of these $\mathrm{SH}$ surfaces exhibit peculiar features like micro- and nano-hierarchical roughness, leading to low surface energy, mimicking $\mathrm{SH}$ surfaces found in Nature, such as the lotus leaf.

Herein, we propose the use of flat $\mathrm{SH}$ substrates to arrange arrays of quasi-spherical cell culture droplets with the capability to build-up 3D spheroids in a high throughput manner, in order to mimic in vivo tumour models on the lab-on-chip scale. Droplets of fibroblasts-like cells (L929) suspension are fixed in defined positions by the action of micro-indentations 
patterned with an array arrangement on polystyrene SH surfaces. The $\mathrm{SH}$ platform is solely physically modified polystyrene (PS), ${ }^{27-33}$ which guarantees the lack of cytotoxicity and the chemical stability of the platform. Such platform presents several advantages compared to the conventional methods used for spheroid formation. Micro-size droplets (volume of $5 \mu \mathrm{L}$ ) for cellular assays allow drastic reduction of the volume required for cell growth, while $\mathrm{SH}$ surfaces mimic the effect of rose petals allowing a minimum contact of the liquid to the surface, avoiding any interference such as protein adsorption or release of molecules from the platform to the cell culture media. The cell culture medium can be changed easily without destroying the micro-tissues since the droplets are tightly linked to the substrates by strong capillary forces, and drugs or other molecules can be easily added at any time. Moreover, the platform is compatible or easily adapted with many analysis techniques, such as inverted confocal microscopy, in which droplets of the cell suspension remain inverted on the $\mathrm{SH}$ surface; and high-throughput combinatorial analysis may be performed in situ using different cell densities or drug concentrations. The high stability, precise positional and adhesiveness of the droplets allow to invert the platform $180^{\circ}$ according to hanging drop approaches. Under this configuration the cells initially suspended in the droplets give rise to spheroids formed by gravity. The innovation of our system relies on the spherical geometry ("hanging spherical drop" method) exhibited by the cell suspension droplets. The size and composition of each droplet can be easily controlled in an individual form.

To explore the capability of this platform, the cell behaviour of $3 \mathrm{D}$ spheroids was compared to $2 \mathrm{D}$ culture upon being subjected to the effect of various concentrations of doxorubicin. Cell viability was evaluated using image-based analysis tools to assess the dose-dependent response of the formed tumour spheroids to this anticancer drug.

A platform was created following biomimetic principles. ${ }^{34}$ Polystyrene (PS) superhydrophobic ( $\mathrm{SH}$ ) substrate mimicking the extreme water repellency of the lotus leaf was patterned with adhesive micro-indentations (Fig. 1A.II) simulating the effect of rose petals. ${ }^{35}$ The low adhesion to droplets on the lotus leaf is caused by the air pockets formed between cell papilla (Cassie-Baxter model) while on the rose petal surface the droplets penetrate into the cuticular folds by capillary forces (Wenzel model). ${ }^{33}$

The developed platform was used as a support for arrays of quasi-spherical droplets of cell suspensions (Fig. 1A.I), for the generation of fibroblast-like cell spheroids. PS sheets were treated by a phase separation methodology, ${ }^{36}$ resulting in a water contact angle (WCA) higher than $150^{\circ}$, mainly by the introduction of micro-nano roughness on the surface (Fig. 1A.III).

We hypothesize that the micro-indentations created on low surface-energy surfaces allow one to confine and stabilize nearly spherical droplets only on the desired places with minimum contact area between the droplet and the surface, even when the surface with cell suspension droplets are turned upside down (Fig. 1A.IV).

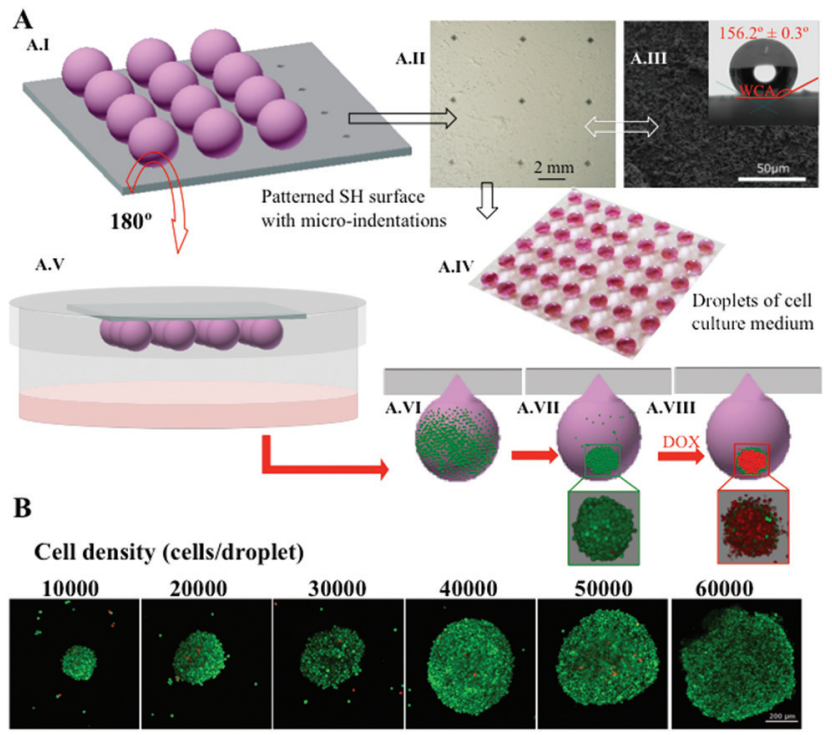

Fig. 1 (A.I) Upper view scheme of the SH platform (A.II) patterned with micro-indentations able to suspend arrays of droplets containing cells. (A.III) The microstructure of PS SH surface observed by SEM and the representative image of a water droplet over the surface. (A.IV) Image of the hanging spherical drops system able to confine droplets on the desired places with minimum contact area between droplets and the surface. (A.V) Turning the platform $180^{\circ}$, (A.VI) the cell suspension inside the droplet (A.VII) leads to the formation of spheroids after 2 days due to the gravitational force applied to the cells. (A.VIII) Drug-screening tests by adding the anti-cancer drug, doxorubicin, in the droplets containing cell spheroids. (B) Live/dead cells assay images after 48 hours of culture were taken using a confocal microscope of the formation of spheroids. Distinct cell densities were tested. Scale bar: $200 \mu \mathrm{m}$.

Video 1 (ESI $\dagger$ ) shows that the platform can be rotated and tilted several times and the droplets of cell culture medium remain fixed with high stability. The volume of droplets used in the micro-indentations can be fixed on the surfaces, without compromising the water repellency of the surface. Increasing the volume deposited in the micro-indentation decreases the sliding angles as the capillary forces exert a less adhesion effect onto bigger droplets. The handling and the movement of the chip, medium exchange and drug delivery to the droplet could be carried out easily, by simply tilting the culture plate lid. The platforms were robust enough allowing successive tilting and the droplets do not slip from the microindentation spots. In this proposed device, the cells are maintained in suspension and are confined within independent miniaturized cell culture droplets surrounded essentially by the gaseous environment of the device. The platform was fixed into the lids of polystyrene petri dishes (commercially available), and the bottom part was filled with cell culture media, in order to create a saturated environment and avoid evaporation of the droplets (Fig. 1A.V-VI). The gravitational force applied on the cells in the hanged droplets induced its sedimentation and the formation of cell spheroids after $48 \mathrm{~h}$ of seeding time (Fig. 1A.VII). Each droplet in the chip forms a singular spheroid with a consistent cell population that is generated without the need of any scaffold or adhesive substances. 


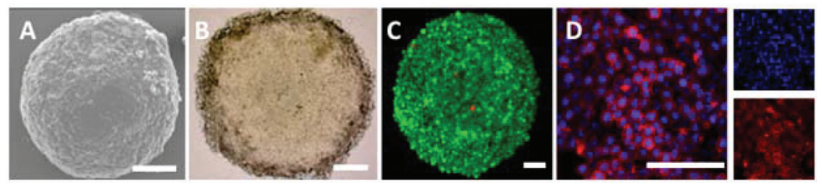

Fig. 2 (A) SEM, (B) bright field, (C) live/dead cells and (D) dapi/phalloidin representative images of $\mathrm{L} 929$ spheroids after 48 hours of culture. Scale bar: $100 \mu \mathrm{m}$.

It was possible to produce arrays of individual sedimented spheroids and test distinct doses of an anti-cancer drug widely used in drug screening, doxorubicin (Dox), in the same device (Fig. 1A.VIII). Conventional methods to produce spheroids have common drawbacks, such as the formation of the spheroids with irregular sizes, inaccessible for testing in the platforms and difficult to adapt to HTS platforms. The proposed hanging spherical drop system allows the growth of homogenously sized spheroids in an easy and non-invasive way.

For the proof-of-concept, droplets containing a fibroblast-like cell suspension were dispensed on the inverted platform in order to promote the spheroid formation. Using distinct cell numbers, spheroids with different sizes and densities were obtained (Fig. 1B). The images of the spheroids were taken using a confocal microscope for precise cell quantification. The stained spheroids with calcein-AM (corresponding to green cells) and propidium iodide (corresponding to red cells) show that after 48 hours of incubation the spheroids were entirely formed with high survival rates. As expected, the diameter of L929 spheroids increased with increasing cell density, from 10000 to 60000 cells perdroplet. Images of spheroids with greater cell densities (from 5000 to 60000 cells per droplet), taken using a fluorescent microscope, can be observed in Fig. S1A and B. $\uparrow$ The micro-tissues produced by the gravity-enforced hanging drop method with a density of 40000 cells per drop present a perfect and uniform integration of single cells into a compact microstructure (Fig. 2A-C).

Spheroids have become a "gold standard" to investigate the cellular signalling mechanisms and effects of anti-tumour agents, mimicking the in vivo situation. Cancer cells live in a complex microenvironment consisting of non-cancer cells and tumour stroma. The tumour stroma consists of the basement membrane, immune cells, ECM, blood vasculature, inflammatory cells and fibroblasts. All are known to contribute to cancer development. Fibroblasts are the major component in the stroma and are responsible for the production of cytokines, ECM and growth factors that promote tumour growth. ${ }^{37}$ Under such conditions, fibroblasts can acquire a myofibroblastic phenotype and make direct cell-cell contact, via gap junction intercellular communication without intervening in the $\mathrm{ECM}^{38-40}$ (Fig. 2D). However, when the fibroblasts are forced to cluster into a spheroid shape, they undergo a new pathway of cell activation beginning a process considered as necrosis-like death, induced by hypoxia or toxins (Fig. 3A.I and Video 2 of the ESI $\dagger$ ). The limited flow of oxygen and nutrients into the core compromises the viability of the fibroblasts; as a result, the
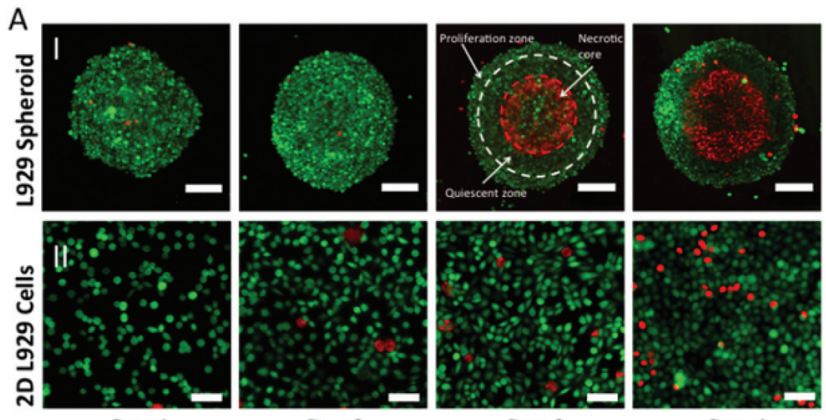

Day 1
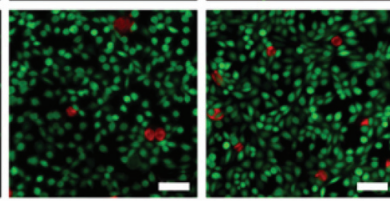

Day 3

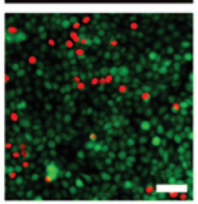

2D Cell Culture

3D Spheroid Culture

B

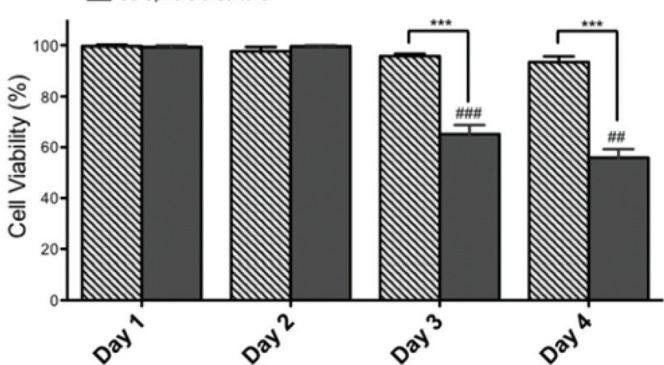

Fig. 3 (A.I) Representative images using live/dead cells staining taken from confocal microscope of individual spheroids up to four days of culture time, with density of 40000 cells per droplet. Scale bar: $200 \mu \mathrm{m}$. (A.II) Representative images of 1929 monolayer cell culture along the same time points and cell density. Images using live/dead cells staining were taken using fluorescent microscope. Scale bar: $50 \mu \mathrm{m}$. (B) Quantification of the viability of the cells in the spheroids and in the $2 \mathrm{D}$ cell cultures. Statistical differences were marked with $\left(^{* * *}\right)$, which represents $p$-value $<0.001$. To evaluate the statistical differences related to the previous time point double (\#\#) and triple symbols (\#\#\#) were used for $p$-value $<0.01$ and $p<0.001$, respectively. All results are presented as mean \pm standard deviation.

viability of the cells decreases with the culture time. For this reason, a typical spheroid has an outer layer of proliferative cells, an internal layer of quiescent cells and hypoxic cells in the core.

The morphogenesis of 2D cell cultures (Fig. 3A.II) was evaluated along with the spheroid culture, as control. In both conditions 40000 cells were used and live/dead cells assay was performed to determine the cell viability. The images of the cluster cells were taken using confocal microscopy, in order to provide accurate quantification of the 3D samples.

The images of $2 \mathrm{D}$ cell culture were taken using a fluorescence microscope. With increasing cell culture time, the cells in 2D monolayer presented higher viability compared to 3D spheroids (Fig. 3B). This can be explained by the fact that cells cultured in monolayer have a higher surface area to adhere and proliferate, whereas 3D spheroids are limited to cell-cell interactions since they are cultured in suspension. Additionally, cells in monolayers have higher access to the nutrients of culture media, compared to the necrotic core of spheroids.

The cellular pathway targeted by a drug, such as doxorubicin (Dox), might differ from 2D to $3 \mathrm{D}$ condition. Dox is an anti-cancer agent widely used in human cancer chemotherapy 
that inhibits RNA and DNA synthesis. ${ }^{41}$ This drug induces an alternative cell death mechanism to apoptosis.

Fig. 4 shows the effect of distinct concentrations of Dox on the cells in spheroids with different densities. Different amounts of Dox were introduced into the droplets to assess the dose-dependent response of the formed tumour spheroids to this anticancer drug. The viability of the cells in the spheroids was measured after 24 hours by a live/dead cells assay (Fig. 4A). As expected, with the increase of the Dox concentration, cell viability of the spheroids decreased. However, L929 spheroids show a greater resistance to Dox compared to the monolayer L929 cultures (Fig. 4B and C).

Recent experiments have confirmed that a tumour spheroid is more resistant to drugs compared with the corresponding

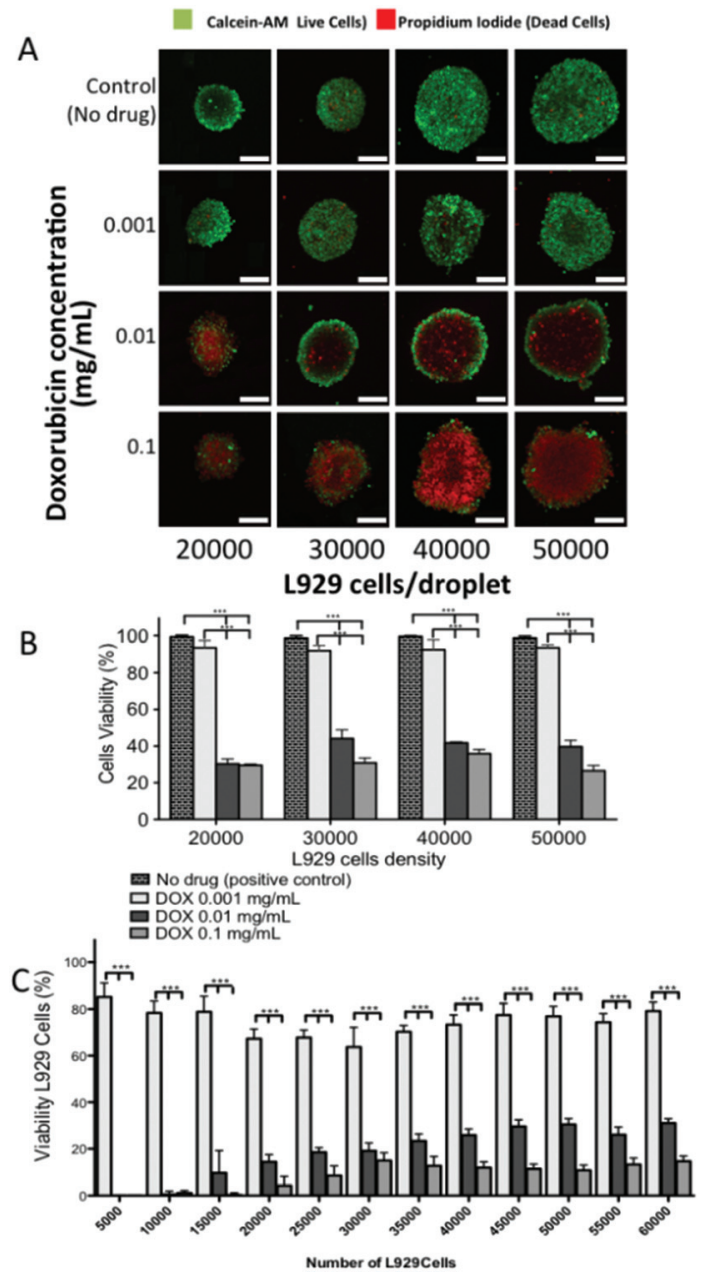

Fig. 4 (A) Representative images of $L 929$ spheroids with distinct densities (after 48 hours of incubation) obtained by using confocal microscopy, after $24 \mathrm{~h}$ of the addition of various concentrations of doxorubicin. Scale bar: $200 \mu \mathrm{m}$. (B) Percentage of viable cells in the 3D spheroids using different cell densities and concentrations of doxorubicin, obtained using imageJ analysis. (C) Cells viability in the 2D cell culture monolayer using different cell densities and concentrations of doxorubicin, obtained by the alamarBlue method. Absorbance was read at wavelength of $570 \mathrm{~nm}$ and $600 \mathrm{~nm}$. Statistical differences were marked ${ }^{(* \star *)} p<0.001$. All results are presented as mean \pm standard deviation.
2D cell cultures. ${ }^{42,43}$ This increased Dox resistance in the spheroid cells may be due to cell-cell contact response, internal protective mechanisms, and/or mass transfer limitations.

Extending the screening of cells in suspension, we employed the new arrangement to test the behaviour of cells in an aggregated form that could be useful for high-throughput monitoring. The proposed methodology could be easily extended to assess the time response of the cells to multiple drugs, drug screening on co-cultures, and study the effect of the gaseous environment on cell behaviour (e.g. hypoxia, normoxia and hyperoxia). In future, it may be possible to adapt the platform for the study of complex cellular spheroids, composed by two or more cell types, in contact with distinct drugs. Multicellular spheroids are widely used to be representative of in vivo avascular tumour regions, and this can represent an opportunity to mimic aspects of native tumour angiogenesis in vitro. Furthermore, the vascularization may be evaluated using these platforms, once spheroids represent the ideal conditions that lead to the self-assembly of tissues mimicking their natural in vivo behaviour.

\section{Conclusions}

A superhydrophobic biomimetic surface based on the socalled lotus effect was produced onto which arrays of microindentations were generated to fix cell suspension droplets, based on the rose petal effect. By facing down the chip, it was possible to produce arrays of cell spheroids developed by gravity in the suspended droplets, according to an hanging spherical drop method. Spheroids provide a 3D cellular environment, which more accurately recapitulates the in vivo tumour, and have been used for drug screening tests. Using this platform, the dose-dependent cytotoxic effect of doxorubicin was measured, and we observed decreasing viability of the cells with increasing doxorubicin concentrations. The spheroids also exhibit enhanced resistance to the drug over $2 \mathrm{D}$ standard monolayer cultures. We believe that such a platform may also have the potential to be used in several biological or biomedical applications, including in the field of tissue engineering and regenerative medicine, drug discovery, and drug delivery monitoring.

\section{Acknowledgements}

The authors thank the precious help of Alessandra Zonari and Rui Domingues for capturing the confocal images. The authors acknowledge the financial support from the FCT- Fundação para a Ciência e para a Tecnologia through the Ph.D. grants with the references SFRH/BD/73119/2010, SFRH/BD/ 69529/2010 and SFRH/BD/71396/2010. We acknowledge the financial support of FEDER through the program Operacional Factores de Competitividade - COMPETE and from FCT - the Fundação para a Ciência e a Tecnologia under the project PTDC/CTM-BIO/1814/2012. We also thank the support by the 
European Research Council grant agreement ERC-2012-ADG 20120216-321266 for the project ComplexiTE.

\section{Notes and references}

1 B. G. Chung, L. F. Kang and A. Khademhosseini, Expert Opin. Drug Discovery, 2007, 2, 1653.

2 S. W. Cranford, J. de Boer, C. van Blitterswijk and M. J. Buehler, Adv. Mater., 2013, 25, 802.

3 A. N. Efremov, E. Stanganello, A. Welle, S. Scholpp and P. A. Levkin, Biomaterials, 2013, 34, 1757.

4 M. Schenone, V. Dancik, B. K. Wagner and P. A. Clemons, Nat. Chem. Biol., 2013, 9, 232.

5 F. Pampaloni, E. G. Reynaud and E. H. K. Stelzer, Nat. Rev. Mol. Cell Biol., 2007, 8, 839.

6 C. J. Torrance, V. Agrawal, B. Vogelstein and K. W. Kinzler, Nat. Biotechnol., 2001, 19, 940.

7 H. M. Dunstan, C. Ludlow, S. Goehle, M. Cronk, P. Szankasi, D. R. H. Evans, J. R. Simon and J. R. Lamb, J. Natl. Cancer Inst., 2002, 94, 88.

8 H. Tian, L. Ip, H. Luo, D. C. Chang and K. Q. Luo, Br. J. Pharmacol., 2007, 150, 321.

9 H. R. Li, H. Y. Zhou, D. Wang, J. S. Qiu, Y. Zhou, X. Q. Li, M. G. Rosenfeld, S. Ding and X. D. Fu, Proc. Natl. Acad. Sci. U. S. A., 2012, 109, 4609.

10 L. F. Willoughby, T. Schlosser, S. A. Manning, J. P. Parisot, I. P. Street, H. E. Richardson, P. O. Humbert and A. M. Brumby, Dis. Models Mech., 2013, 6, 521.

11 E. Atefi, S. Lemmo, D. Fyffe, G. Luker and H. Tavana, Adv. Funct. Mater., 2014, 24, 6509.

12 S. Lemmo, E. Atefi, G. Luker and H. Tavana, Cell. Mol. Bioeng., 2014, 7, 344.

13 M. T. Santini, G. Rainaldi and P. L. Indovina, Crit. Rev. Oncol. Hematol., 2000, 36, 75.

14 L. G. Griffith and M. A. Swartz, Nat. Rev. Mol. Cell Biol., 2006, 7, 211.

15 K. M. Yamada and E. Cukierman, Cell, 2007, 130, 601.

16 M. Rimann and U. Graf-Hausner, Curr. Opin. Biotechnol., 2012, 23, 803.

17 E. Fennema, N. Rivron, J. Rouwkema, C. van Blitterswijk and J. de Boer, Trends Biotechnol., 2013, 31, 108.

18 E. W. K. Young, Integr. Biol., 2013, 5, 1096.

19 J. Friedrich, C. Seidel, R. Ebner and L. A. Kunz-Schughart, Nat. Protoc., 2009, 4, 309.

20 R. Z. Lin and H. Y. Chang, Biotechnol. J., 2008, 3, 1172.
21 Y. C. Tung, A. Y. Hsiao, S. G. Allen, Y. S. Torisawa, M. Ho and S. Takayama, Analyst, 2011, 136, 473.

22 G. M. Luz, A. J. Leite, A. I. Neto, W. L. Song and J. F. Mano, Mater. Lett., 2011, 65, 296.

23 A. I. Neto, C. A. Custodio, W. L. Song and J. F. Mano, Soft Matter, 2011, 7, 4147.

24 C. L. Salgado, M. B. Oliveira and J. F. Mano, Integr. Biol., 2012, 4, 318.

25 M. B. Oliveira and J. F. Mano, Anal. Chem., 2013, 85, 2391.

26 M. B. Oliveira, C. L. Salgado, W. L. Song and J. F. Mano, Small, 2013, 9, 768.

27 W. L. Song, A. C. Lima and J. F. Mano, Soft Matter, 2010, 6, 5868.

28 A. C. Lima, W. Song, B. Blanco-Fernandez, C. AlvarezLorenzo and J. F. Mano, Pharm. Res., 2011, 28, 1294.

29 S. M. Oliveira, W. L. Song, N. M. Alves and J. F. Mano, Soft Matter, 2011, 7, 8932.

30 A. C. Lima, P. Sher and J. F. Mano, Expert Opin. Drug Delivery, 2012, 9, 231.

31 A. C. Lima, C. A. Custodio, C. Alvarez-Lorenzo and J. F. Mano, Small, 2013, 9, 2487.

32 A. C. Lima, C. R. Correia, M. B. Oliveira and J. F. Mano, J. Bioact. Compat. Polym., 2014, 29, 50.

33 S. M. Oliveira, N. M. Alves and J. F. Mano, J. Adhes. Sci. Technol., 2014, 28, 843.

34 A. I. Neto, H. J. Meredith, C. L. Jenkins, J. J. Wilker and J. F. Mano, RSC Adv., 2013, 3, 9352.

35 Z. J. Cheng, M. Du, H. Lai, N. Q. Zhang and K. N. Sun, Nanoscale, 2013, 5, 2776.

36 N. M. Oliveira, A. I. Neto, W. L. Song and J. F. Mano, Appl. Phys. Express, 2010, 3, 085205.

37 K. Rasanen and A. Vaheri, Exp. Cell Res., 2010, 316, 2713.

38 S. Yonemura, M. Itoh, A. Nagafuchi and S. Tsukita, J. Cell Sci., 1995, 108, 127.

39 S. G. Spanakis, S. Petridou and S. K. Masur, Invest. Ophthalmol. Vis. Sci., 1998, 39, 1320.

40 V. A. Krutovskikh, C. Piccoli and H. Yamasaki, Oncogene, 2002, 21, 1989.

41 G. Aubelsadron and D. Londosgagliardi, Biochimie, 1984, 66, 333.

42 L. F. Yu, M. C. W. Chen and K. C. Cheung, Lab Chip, 2010, 10, 2424.

43 I. Amjadi, M. Rabiee, M. S. Hosseini and M. Mozafari, Appl. Biochem. Biotechnol., 2012, 168, 1434. 